\title{
Prevalence of Early and Late Age-Related Macular Degeneration in a Rural Population in Northern India: The INDEYE Feasibility Study
}

\author{
Sanjeev K. Gupta, ${ }^{1}$ Gudlavalleti V. S. Murtby, ${ }^{1}$ Nicoli Morrison, ${ }^{2}$ Gill M. Price, ${ }^{3}$ \\ Mukesh Dherani, ${ }^{3}$ Neena Jobn, ${ }^{1}$ Astrid E. Fletcher, ${ }^{3}$ and Usha Chakravarthy ${ }^{2}$
}

Purpose. To assess the prevalence of age-related macular degeneration (AMD) in a rural population in Northern India.

Methods. In a pilot feasibility study, 1443 people (median age, 60 years; $52 \%$ women), were identified from enumeration of the $50+$ age group in 11 randomly sampled villages from a rural, periurban district of Haryana, Northern India. Of those identified, $87 \%$ attended an eye examination that included digital fundus photography. Fundus images were graded at a single reading center using definitions from the Wisconsin Age-Related Maculopathy Grading System.

Results. Fundus photographs were available for 1101 participants. Overall, $28.8 \%$ of participants had ungradable fundus images due to cataract. Including all with ungradable images in the denominator, the prevalence of soft drusen was $34.0 \%$ (95\% confidence interval [CI] 26.1-42.9); of soft indistinct drusen, 2.2\% (95\% CI, 1.1-4.4); and of pigmentary irregularities, $10.8 \%$ (95\% CI, 7.1-16.1). There were 15 (1.4\%) cases of late-stage AMD (95\% CI, 0.8-2.3) with the prevalence rising from $0.4 \%$ in the 50 - to 59 -year age range to $4.6 \%$ in those aged 70 years or older.

Conclusions. Drusen and pigmentary irregularities are common among the rural northern Indian population. The prevalence of late AMD is similar to that encountered in Western settings and is likely to contribute significantly to the burden of vision loss in older people in the developing world. (Invest Ophthalmol Vis Sci. 2007;48:1007-1011) DOI:10.1167/iovs.060712

T $\mathrm{n}$ developed countries, the advanced manifestations of agerelated macular degeneration (AMD) are the main cause of adult blindness. ${ }^{1}$ Although there is considerable information on visual impairment and blindness in the developing world, to date there are few data on the prevalence of early or advanced AMD outside Western settings. ${ }^{2-3}$ Two recent studies from Southern India suggest that AMD may be as commonly encoun-

From the ${ }^{1}$ Department of Community Ophthalmology, Dr Rajendra Prasad Centre for Ophthalmic Sciences, All India Institute of Medical Sciences, New Delhi, India; ${ }^{2}$ Ophthalmology and Vision Science, The Queen's University of Belfast, Northern Ireland, United Kingdom; and the ${ }^{3}$ Department of Epidemiology and Population Health, London School of Hygiene and Tropical Medicine, London, United Kingdom.

Supported by Wellcome Trust Grant 066082 .

Submitted for publication June 26, 2006; revised August 18 and November 6, 2006; accepted January 18, 2007.

Disclosure: S.K. Gupta, None; G.V.S. Murthy, None; N. Morrison, None; G.M. Price, None; M. Dherani, None; N. John, None; A.E. Fletcher, None; U. Chakravarthy, None

The publication costs of this article were defrayed in part by page charge payment. This article must therefore be marked "advertisement" in accordance with 18 U.S.C. $\$ 1734$ solely to indicate this fact.

Corresponding author: Astrid E. Fletcher, Department of Epidemiology and Population Health, London School of Hygiene and Tropical Medicine, Keppel St., London WC1E 7HT, UK;

astrid.fletcher@1shtm.ac.uk. tered as it is in Western countries. ${ }^{4-5}$ It is imperative therefore that in countries with rapidly growing older populations, estimates of the prevalence of AMD be obtained, to enable planning for low-vision services and rehabilitation. The body of evidence from studies of Western populations implicate lifestyle-related, nutritional, and genetic risk factors in the pathogenesis of AMD. ${ }^{6}$ However, the interactions between these risk factors and the biochemical, cellular, and molecular pathways that influence its development are unknown. Age is the major risk factor for AMD and it is possible that this disease may become more important in countries undergoing population ageing. The demographic profile of India is changing, with rapid increases in the older population. An approximately 2.5-fold increase in those aged 60 years and over is projected between 2001 ( 71 million at 2001 census) and 2031. ${ }^{7}$ However, the Indian subcontinent has large rural areas where the population have a lifestyle and diet that differ markedly from those in Western countries. A study of the prevalence of AMD along with an investigation of putative risk factors-principally environment, lifestyle, and diet-in a rural population in India would yield information, not only for provision of eye health services, but also for additional insights into the pathogenesis of the disorder.

The present study was therefore undertaken as a pilot feasibility study to obtain prevalence estimates of early and advanced AMD.

\section{MeTHODS}

\section{Study Population}

The India Eye Study (INDEYE), a feasibility study, was a single-center survey of persons living in the rural periurban areas of Faridabad district, which is a geographically distinct area with a homogeneous and stable population in Haryana, Northern India. A list of 25 villages was obtained from the 1991 census data (2001 census data were not available at that time), and 11 villages were randomly selected on the basis that $13 \%$ of the population would be 50 years of age or older. Before enumeration, meetings were held with local village leaders to explain the study objectives and methods. In the region, 1443 people (median age, 60 years; $52 \%$ women), were identified from enumeration of the $50+$ age group in 11 villages and invited to take part in the study. Recruitment into the study was performed in the 4-month period between September 2002 and January 2003. Participants who were illiterate had the information leaflet read to them, and subjects were enrolled into the study only after informed written consent (for illiterate participants, this consisted of a thumb impression) was obtained. The study adhered to the guidelines in the Declaration of Helsinki and received ethics approval from the Research Ethics Committees of the All India Institute of Medical Sciences, the London School of Hygiene and Tropical Medicine, and The Queen's University of Belfast.

\section{Ophthalmic Procedures}

Visual acuity (VA) was tested with the tumbling-E ETDRS (Early Treatment Diabetic Retinopathy Study) chart and recorded in each eye 
separately with the subject wearing the habitual spectacles (if any). If VA in either of the two eyes of a participant was worse than logMAR 0.6 , refraction was performed with an autorefractor (Nikon, Tokyo, Japan), and best corrected acuity was recorded. Pupillary dilation was performed using $1 \%$ tropicamide after anterior segment biomicroscopy. A clinical examination of each eye was performed which included anterior and posterior segment assessments with slit lamp biomicroscopy.

Lens photography was performed with a cataract screener (Neitz Instruments Co., Ltd., Tokyo, Japan; SL7E slit lamp; Topcon, Tokyo, Japan) and graded according to Lens Opacities Classification System II (LOCS II). ${ }^{8}$ Fundus photography was undertaken with a fundus acquisition system (TRC $50 \mathrm{EX}$; Topcon) equipped with a high-resolution CCD color digital camera ( 2 million pixels; Sony 3 ; Sony, Tokyo, Japan) and used to photograph the macular retina. The fundus photographic protocol included the capture of a single image of the anterior segment of each eye focused on the lens. Two $35^{\circ}$ stereo photographs of standard field 2 (based on the Wisconsin Age-Related Maculopathy Grading System; WARMGS), ${ }^{9}$ were obtained from each eye. Images were saved to compact disks and mailed to the grading center in Belfast.

Two on-site study personnel who had undergone extensive training in study-related procedures performed acuity testing, refraction when necessary and photography of lens and fundus on study participants who attended the study center. Some participants who could not attend the study center due to ill health were assessed in a home visit during which visual acuity testing and ophthalmic examination were performed $(n=63)$. Fundus images were unavailable in these participants.

\section{Grading of Images}

After images were checked for completeness (availability of stereo pairs from the macula of each eye) and quality (clarity, focus, and stereoscopy) of images, grading of age related maculopathy (ARM) was undertaken based on the Wisconsin Age-Related Maculopathy Grading System. ${ }^{9}$ In this classification, drusen are categorized based on their uniformity of color and margins. Drusen with a uniform density, sharp margins, and a nodular surface texture were placed in the soft distinct category, whereas those without a nodular texture were placed in the hard category. Drusen with decreasing density from the center with blurred margins were placed in the soft, indistinct category. Interlacing defined or ill-defined networks of drusen were placed in the reticular category. Retinal depigmentation was defined as changes in fundus coloration that were inadequate to permit classification as geographic atrophy (GA). Hyperpigmentation was said to be present if focal clumps of increased pigment (brown or black) were found within the retina. Any combination of these photographic features was referred to as early age-related maculopathy. Neovascular AMD was diagnosed when exudative features or GA was present. When any combination of serous fluid, hemorrhage, lipid exudates or fibrosis were seen to be originating primarily from the subretinal and pigment epithelial tissue layers, choroidal neovascularization was considered to be present. GA was defined as the presence of well-demarcated regions with diameters in excess of $175 \mu \mathrm{m}$, within which large choroidal vessels were clearly visible, owing to the atrophy of the overlying choriocapillaris and retinal pigmented epithelium.

A grader (NM) trained in the recognition of photographic signs of ARM performed the grading on all the INDEYE dataset. Suitable pairs of images of the macula (field 2) were displayed on screen for stereo viewing. A grid with a radius corresponding to $3000 \mu \mathrm{m}$ at the retina was placed on one of the stereoscopic pair centered on the geometric center of the fovea. Images underwent detailed grading if the area covered by the grid in field 2 was visible and if ARM features could be clearly distinguished. The predominant drusen type was recorded as well as the number of drusen present within the grid area. In addition the diameter of the largest druse present was noted by using the graded categorical approach recommended by WARMGS (the three
TABLE 1. Five Mutually Exclusive Grades of Age-Related Maculopathy ${ }^{10}$

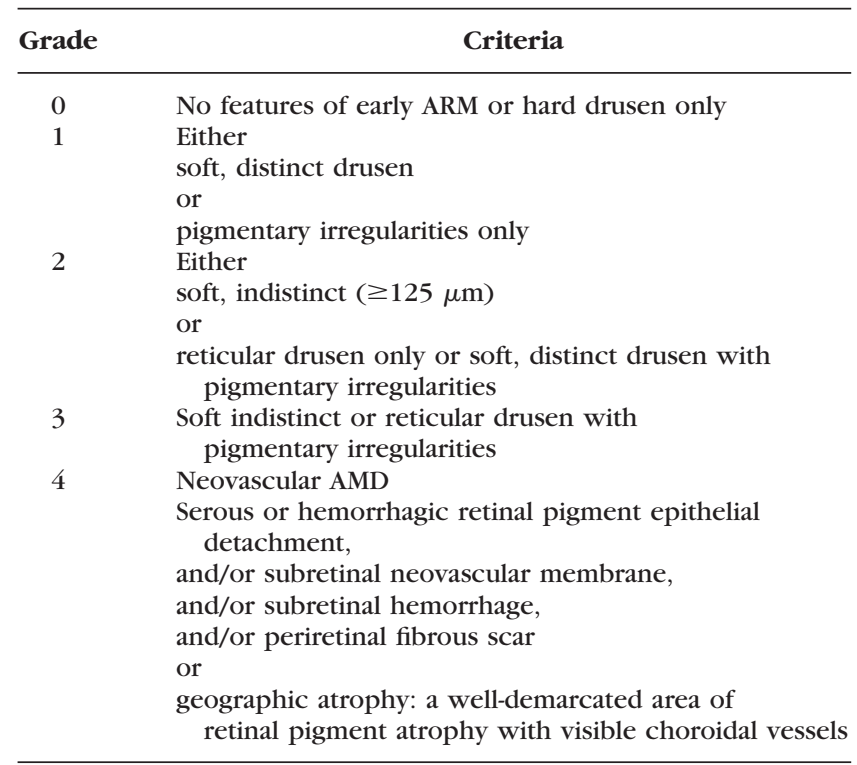

diameter categories were $<63 \mu \mathrm{m}, \geq 63-<125 \mu \mathrm{m}$, and $\geq 125-250$ $\mu \mathrm{m})$. The presence of hypo- and hyperpigmentation was assessed. Subjects were assigned to one of five stages of severity, based on the staging system developed by the investigators of the Rotterdam Eye Study. ${ }^{10}$ This staging system facilitates analysis by compressing the large number of variables measured during the grading of the fundus photographs into one of five mutually exclusive stages (Table 1). All questionable lesions and all eyes classified as having late-stage AMD were adjudicated by the principal investigator (UC). Any lesions considered to be due to other causes such as myopia and inflammatory disease were excluded.

\section{Statistical Analysis}

Cluster (i.e., village) sampling was taken into account in all analyses by "survey" estimation (STATA ver. 8). ${ }^{11}$ Differences in response rates to the clinical examination between men and women and by 10 -year age groups were tested with design-based Pearson tests. Prevalence (\%) was calculated by using data on the eye with more severe features of ARM. Age- (10-year age groups) and gender-specific prevalences and their corresponding 95\% confidence intervals [CIs] were estimated for early features and stage of ARM. Poisson regression and design-adjusted Wald tests were used to estimate effects of age group adjusted for sex and for sex adjusted for age group on the prevalence of ARM (Rotterdam staging). Multivariate logistic regression was used to examine factors (age, sex, and cataract presence) associated with the images of both eyes being classified as "ungradable."

\section{Results}

After enumeration, 1443 potential participants (693 men and 750 women) were identified who were 50 years of age and older. Only four of the sample were ineligible (three died between enumeration and study inception, and one moved from the village). Of the 1439 eligible participants, 1260 (87.6\%) underwent a clinical examination; and of those, 1101 completed all study procedures including dilated fundus photography. Fundus images were not available on 159 participants who had a clinical examination; and of those, 63 were examined at home where photography was not possible. In the remainder, corneal opacities, phthisis bulbi, small pupils, and medical contraindications to pupillary dilation constituted the principal reasons for lack of photographs. There was a slightly 
TABLE 2. Number, Prevalence, and 95\% CI of Features of Age-Related Maculopathy

\begin{tabular}{|c|c|c|c|c|c|c|}
\hline All Ages* & No ARM & $\begin{array}{c}\text { Hard } \\
\text { Drusen }\end{array}$ & $\begin{array}{l}\text { Soft Distinct } \\
\text { Drusen }\end{array}$ & $\begin{array}{c}\text { Soft } \\
\text { Indistinct/Reticular }\end{array}$ & $\begin{array}{l}\text { Pigmentary } \\
\text { Irregularities }\end{array}$ & $\begin{array}{c}\text { Bilateral } \\
\text { Ungradable } \\
\text { Photos }\end{array}$ \\
\hline Men & 20 & 119 & 186 & 17 & 63 & 153 \\
\hline$(n=529)$ & $\begin{array}{c}3.8 \\
1.4-9.7\end{array}$ & $\begin{array}{c}22.5 \\
14.4-33.3\end{array}$ & $\begin{array}{c}35.2 \\
26.8-44.6\end{array}$ & $\begin{array}{c}3.2 \\
1.6-6.4\end{array}$ & $\begin{array}{c}11.9 \\
7.5-18.4\end{array}$ & $\begin{array}{c}28.9 \\
21.7-37.4\end{array}$ \\
\hline $\begin{array}{l}\text { Women } \\
\qquad(n=572)\end{array}$ & $\begin{array}{c}31 \\
5.4 \\
2.1-13.1\end{array}$ & $\begin{array}{c}149 \\
26.0 \\
18.8-34.8\end{array}$ & $\begin{array}{c}188 \\
32.9 \\
25.2-41.5\end{array}$ & $\begin{array}{c}7 \\
1.2 \\
0.5-3.2\end{array}$ & $\begin{array}{c}56 \\
9.8 \\
6.3-15.0\end{array}$ & $\begin{array}{c}164 \\
28.7 \\
21.9-36.5\end{array}$ \\
\hline Age group (y) & & & & & & \\
\hline $\begin{array}{l}50-59 \\
\quad(n=511)\end{array}$ & $\begin{array}{c}26 \\
5.1 \\
1.8-13.5\end{array}$ & $\begin{array}{c}169 \\
33.1 \\
24.9-42.4\end{array}$ & $\begin{array}{c}222 \\
43.4 \\
32.3-55.3\end{array}$ & $\begin{array}{c}14 \\
2.7 \\
1.3-5.8\end{array}$ & $\begin{array}{c}66 \\
12.9 \\
7.6-21.2\end{array}$ & $\begin{array}{c}50 \\
9.8 \\
6.1-15.4\end{array}$ \\
\hline $\begin{array}{l}60-69 \\
\quad(n=352)\end{array}$ & $\begin{array}{c}15 \\
4.3 \\
1.7-10.2\end{array}$ & $\begin{array}{c}76 \\
21.6 \\
13.2-33.3\end{array}$ & $\begin{array}{c}114 \\
32.4 \\
23.5-42.8\end{array}$ & $\begin{array}{c}5 \\
1.4 \\
0.7-2.8\end{array}$ & $\begin{array}{c}42 \\
11.9 \\
8.0-17.5\end{array}$ & $\begin{array}{c}118 \\
33.5 \\
24.1-44.4\end{array}$ \\
\hline $\begin{array}{l}70-79 \\
\quad(n=238)\end{array}$ & $\begin{array}{c}10 \\
4.2 \\
1.1-14.9\end{array}$ & $\begin{array}{c}23 \\
9.7 \\
5.3-17.1\end{array}$ & $\begin{array}{c}38 \\
16.0 \\
10.1-24.4\end{array}$ & $\begin{array}{c}5 \\
2.1 \\
0.4-9.6\end{array}$ & $\begin{array}{c}11 \\
4.6 \\
2.6-8.1\end{array}$ & $\begin{array}{c}149 \\
62.6 \\
53.3-71.1\end{array}$ \\
\hline $\begin{array}{l}\text { Total } \\
\qquad(n=1101)\end{array}$ & $\begin{array}{c}51 \\
4.6 \\
1.8-11.4\end{array}$ & $\begin{array}{c}268 \\
24.3 \\
17.1-33.5\end{array}$ & $\begin{array}{c}374 \\
34.0 \\
26.1-42.9\end{array}$ & $\begin{array}{c}24 \\
2.2 \\
1.1-4.4\end{array}$ & $\begin{array}{c}119 \\
10.8 \\
7.1-16.1\end{array}$ & $\begin{array}{c}317 \\
28.8 \\
22.3-36.2\end{array}$ \\
\hline
\end{tabular}

* 317 participants with both eyes ungradable are included in the denominator.

lower rate of participation in the clinical examination in the men $(85.0 \%)$ than in the women $(89.5 \% ; P=0.054)$. Participation rates were high across all age groups ranging from $86.1 \%$ aged 50 to $59,87.9 \%$ aged 60 to 69 , and $88.7 \%$ aged $70+$ years ( $P=0.4$ for differences between age groups).

In a high proportion of participants with fundus photographs $(28.8 \%)$, images of both eyes were unsuitable for grading (Table 2). People with bilateral ungradable images were more likely to be in the older age groups, Odds ratio (OR) for the age group 70 to 79 years compared with the age group 50 to 59 years was 11.3 (95\% CI, 6.5-19.7). There was no difference by sex in the proportion of ungradable photographs. Fundus photographs of people with cataract (defined as LOCS II grade of 2 or more for nuclear, cortical, or posterior subcapsular opacities or with dense opacities that could not be graded by type) were more likely to be classified as ungradable $(\mathrm{OR}=$
12.0; 95\% CI, 5.5-25.6). Prevalence figures are based on the eye with more severe status if both eyes were gradable and on the gradable eye if only one eye was gradable. People with both eyes ungradable are included in the denominator of early features of ARM and by stage of ARM (Table 3). The prevalence of drusen and pigmentary irregularities by gender and age are shown in Table 2. Drusen were present in a large proportion of the population with small hard and soft distinct being the commonest drusen types encountered.

On classification of participants using the mutually exclusive Rotterdam Eye Study staging system (Table 3), men were more likely than women to be graded as ARM $(P=0.03)$. There was a negative trend by age group for ARM 0 , ARM 1, and ARM 2, whereas increasing age was associated with AMD $(P<0.05)$.

TABLE 3. Number, Prevalence, and 95\% CI of Grades of Age-Related Maculopathy, as Defined in Table 1

\begin{tabular}{|c|c|c|c|c|c|}
\hline All Ages* & No ARM & ARM 1 & ARM 2 & ARM 3 & AMD \\
\hline Men & 139 & 181 & 36 & 10 & 10 \\
\hline \multirow[t]{2}{*}{$(n=529)$} & 26.3 & 34.2 & 6.8 & 1.9 & 1.9 \\
\hline & $15.5-40.8$ & $25.9-43.6$ & $4.7-9.8$ & $0.9-4.1$ & $1.1-3.2$ \\
\hline Women & 180 & 192 & 27 & 4 & 5 \\
\hline \multirow[t]{2}{*}{$(n=572)$} & 31.5 & 33.6 & 4.7 & 0.7 & 0.9 \\
\hline & $20.8-44.6$ & $26.8-41.1$ & $3.1-7.0$ & $0.3-1.8$ & $0.3-2.4$ \\
\hline$P$, effect of sex & 0.3 & 0.2 & 0.08 & 0.03 & 0.3 \\
\hline \multicolumn{6}{|l|}{ Age group (y) } \\
\hline $50-59$ & 195 & 220 & 36 & 8 & 2 \\
\hline \multirow{2}{*}{$(n=511)$} & 38.2 & 43.1 & 7.0 & 1.6 & 0.4 \\
\hline & $26.1-51.9$ & $33.7-53.0$ & $5.2-9.4$ & $0.7-3.7$ & $0.0-4.0$ \\
\hline $60-69$ & 91 & 120 & 17 & 4 & 2 \\
\hline \multirow[t]{2}{*}{$(n=352)$} & 25.9 & 34.1 & 4.8 & 1.1 & 0.6 \\
\hline & $15.4-40.1$ & $24.5-45.2$ & $2.7-8.4$ & $0.5-2.7$ & $0.1-2.2$ \\
\hline $70-79$ & 33 & 33 & 10 & 2 & 11 \\
\hline \multirow[t]{2}{*}{$(n=238)$} & 13.9 & 13.9 & 4.2 & 0.8 & 4.6 \\
\hline & $7.0-25.6$ & $9.4-19.9$ & $2.0-8.5$ & $0.1-7.5$ & $2.7-7.8$ \\
\hline$P$, trend by age group & 0.0002 & $<0.00001$ & 0.03 & 0.3 & 0.04 \\
\hline Total & 319 & 373 & 63 & 14 & 15 \\
\hline \multirow[t]{2}{*}{$(n=1101)$} & 29.0 & 33.9 & 5.7 & 1.3 & 1.4 \\
\hline & $18.5-42.3$ & $26.6-42.0$ & $4.2-7.8$ & $0.6-2.6$ & $0.8-2.3$ \\
\hline
\end{tabular}

\footnotetext{
*317 participants with both eyes ungradable are included in the denominator.
} 
The overall prevalence of advanced AMD in the INDEYE study population was $1.4 \%$ (95\% CI, $0.8-2.3)$. There was only one case of GA with choroidal neovascularization accounting for all the remainder. All 15 cases with advanced AMD were visually impaired, with best corrected acuity in the betterseeing eye worse than 20/80 Snellen.

\section{Discussion}

This is the first study in the Indian subcontinent to report on the prevalence of the full spectrum of ARM based on fundus photography and internationally accepted grading systems. There are sparse data on the prevalence of ARM outside Western countries, although studies in the United States have examined the prevalence of ARM by ethnic group, predominantly African Americans and Hispanic Americans. Several studies have found the prevalence of AMD is extremely low in African Americans or African Caribbeans compared with European or European origin populations. ${ }^{12-15}$ In a pooled analysis of the major population based studies in Europe, the United States, and Barbados, the rates for African American participants were around a third of those of participants of European origin and around a tenth in the oldest age group. ${ }^{16}$ For Hispanic Americans the results from studies are less consistent. The largest study, the Los Angeles Latino Eye Study (LALES) ${ }^{17}$ found that rates of AMD were comparable to those in populations of European origin but the Proyecto Ver study ${ }^{18}$ reported lower rates than did LALES. In the NHANES III (National Health and Nutrition Examination Survey) population, rates of AMD in Mexican Americans were lower than those of non-Hispanic whites $^{13,15}$ but early ARM (defined as drusen and pigmentary irregularities) was of a similar prevalence in non-Hispanic blacks, non-Hispanic whites, and Mexican-Americans. ${ }^{15}$ The Baltimore Eye Survey ${ }^{14}$ found that small, hard drusen were equally prevalent in white and black subjects; however, large drusen $(>125 \mu \mathrm{m})$ occurred more frequently among whites than blacks, and this difference was particularly noticeable in the age group above 70 years. The finding of a lower prevalence of large drusen in African Americans was also shown in the pooled analysis of the major population studies. ${ }^{16}$ The LALES study reported even higher rates of large drusen compared with the rates reported for European Americans or African Americans. ${ }^{17}$ The prevalence of soft, distinct or pigmentary irregularities (ARM grade 1) in INDEYE participants was similar to that reported in the seven-country EUREYE study which used similar methods of fundus photography and grading to those used in INDEYE. ${ }^{19}$ In EUREYE, 36.5\% of the $65+$ population had ARM1 compared with $33.9 \%$ in the INDEYE population, despite its lower age. For stage 2 (large, soft, indistinct drusen or soft, distinct drusen with pigmentary irregularities) the figures were $10.1 \%$ and $5.7 \%$, respectively. Whereas the finding of differences in the present study may represent real racial variation in the frequencies in which the features of early ARM occur in populations, the high percentage of cataract precluding assessment of a significant number of the fundus images in the older Indian participants may have resulted in an underestimation of the prevalence of the more severe drusen types that normally increase with increasing age.

Very recently, two studies have reported the prevalence of AMD in the Indian subcontinent. ${ }^{4-5}$ The Aravind Comprehensive Eye Study of people aged 40 years and more, undertaken in the state of Tamil Nadu in Southern India, ${ }^{4}$ reported the prevalence of vitreoretinal disorders including AMD. In that study, early AMD (defined as the presence of soft large drusen greater than $125 \mu \mathrm{m}$ in diameter with pigment epithelial abnormalities) was found in $2.7 \%$ of the population. This definition of early AMD corresponds to stage 3 of the Rotterdam staging system, and we found a prevalence of $2.0 \%$ in the INDEYE population. Thus, there was a high degree of similarity despite the use of different methods of AMD detection (slit lamp biomicroscopy in the Aravind Survey versus digital image grading in INDEYE). The prevalence of late AMD (defined as the presence of exudative AMD or GA) was $0.6 \%$ in the Aravind Eye Survey, whereas it was higher in the present study (1.6\%). This difference may be explained by the older age of the population surveyed in INDEYE study, but it is worth noting that the prevalence of late AMD in the Aravind Survey rose from $1.3 \%$ in the 40 - to 49 -year age range to $6.9 \%$ in those aged $\geq 70$ years

The more recently published Andhra Pradesh Eye Study (APEDS) reported only the prevalence of advanced AMD, although information was collected through photographic grading on early features including hard and soft drusen, hyper- and hypopigmentation. ${ }^{5}$ APEDS sampled a population aged $\geq 40$ years and there were 71 cases of late AMD. This number represented an overall prevalence of $1.9 \%$, rising from $0.9 \%$ in the 40 - to 49 -year age group to $3.7 \%$ in the $\geq 70$ age group. No information was provided on the proportion of ungradable eyes, even though $39 \%$ of the sample was reported to have cataract. It was also notable that an overwhelming majority (94\%) of advanced AMD cases were classified as dry advanced AMD (that is GA). Although this is in accord with the findings of the Aravind Comprehensive Survey, ${ }^{4}$ the higher ratio of GA to exudative AMD was not observed in the Northern Indian population sampled in INDEYE. However, the findings of the present study are more in accord with other population-based studies, where exudative AMD is detected twice as often as $\mathrm{GA}^{16}$

The INDEYE Study acquired high-resolution digital images of the macular retina for grading. These images were reviewable on screen, permitting real-time assessment of fundus details and photographic quality at the time of image acquisition. The EUREYE study previously validated the use of digital images by comparing them against concurrently acquired analogue images captured on $35-\mathrm{mm}$ film. ${ }^{20}$ In fact, fewer than $3 \%$ of images from participants in EUREYE were pronounced ungradable. ${ }^{19}$ Nonetheless, despite the use of digital imaging technology in the INDEYE study, images from just under a third of all participants were ungradable, owing to the high prevalence of cataract. As both AMD and cataract increase in prevalence with ageing, it is likely that the medial opacities in eyes of older participants in INDEYE has led to an underestimation of the true prevalence of drusen and pigmentary irregularities.

In the present study, the prevalence of neovascular AMD was closer to rates for European populations and higher than those for African Americans and African Caribbeans. ${ }^{16}$ However, the relatively small sample size of the present study precludes definitive estimates of the prevalence of AMD. A larger study of 6000 participants older than 60 years in two centers in India is now under way and will provide more precise estimates of the prevalence of early and late manifestations of ARM in two geographically distinct locations within the Indian subcontinent.

\section{Acknowledgments}

The authors thank Vittorio Silvestri (Photographer, Royal Hospitals, Belfast) for provision of training to the INDEYE study team; Mike Neider (Fundus Photograph Reading Center, Wisconsin), for his advice and assistance in establishing the photographic protocols; Ada Hooghart (Rotterdam Eye Study Fundus Photographic Grading Centre) for help with the training of Nicoli Morrison; and the study team, Tara D. Pant, Rehabilitation Assistant and Survey Field Supervisor, Lalit Sanga, Survey Ophthalmologist, and Hira Pant and Kurishummutil G. Verghese, data entry operators. 


\section{References}

1. Thylefors B, Negrel AD, Pararajasegaram R, Dadzie KY. Global data on blindness. Bull World Health Organ. 1995;73:115-121.

2. West SK, Sommer A. Prevention of blindness and priorities for the future. Bull World Health Organ. 2001;79:244-248.

3. West SK. Looking forward to 20/20: a focus on the epidemiology of eye diseases. Epidemiol Rev. 2000;22:64-70.

4. Nirmalan PK, Katz J, Robin AL, et al. Prevalence of vitreo retinal disorders in a rural population of Southern India: The Aravind Comprehensive Eye Study. Arch Opbthalmol. 2004;122:581-586.

5. Krishnaiah S, Das T, Nirmalan PK, et al. Risk factors for age-related macular degeneration: findings from the Andhra Pradesh eye disease study in South India. Invest Ophthalmol Vis Sci. 2005;46: 442-449.

6. Smith W, Assink J, Klein R, et al. Risk factors for age-related macular degeneration: pooled findings from three continents. Opbthalmology. 2001;108:697-704.

7. Rajan SI, Sarma PS, Mishra US. Demography of Indian aging, 20012051. J Aging Soc Policy. 2003;15:11-30.

8. Chylack LT Jr, Leske MC, McCarthy D, Khu P, Kashiwagi T, Sperduto R. Lens opacities classification system II (LOCS II). Arch Opbthalmol. 1989;107:991-997.

9. Klein R, Davis M, Magli Y, Segal P, Klein B, Hubbard L. The Wisconsin Age Related Maculopathy Grading System. Opbthalmology. 1991;98:1128-1134.

10. Klaver CCW, Assink JJM, van Leeuwen R, et al. Incidence and progression rates of age-related maculopathy: The Rotterdam Study. Invest Ophthalmol Vis Sci. 2001;42:2237-2241.

11. Stata Statistical Software: Release 8.0. 2003. College Station, TX: Stata Corp. 2003.
12. Schachat A. Features of age-related macular degeneration in a black population: The Barbados Eye Study Group. Arch Ophthalmol. $1995 ; 113: 728-735$.

13. Klein R, Rowland ML, Harris MI. Racial/Ethnic differences in agerelated maculopathy: third National Health and Nutrition Survey. Opbthalmology. 1995;102:371-381.

14. Friedman DS, Katz J. Bressler NM, Rahmani B, Tielsch JM. Racial differences in the prevalence of age-related macular degeneration: the Baltimore Eye Survey. Opbthalmology. 1999;106:1049-1055.

15. Klein R, Klein BE, Jensen SC, et al. Age-related maculopathy in a multiracial United States Population: the National Health and Nutrition Examination Survey III. Ophthalmology. 1999;106:10561065.

16. Friedman DS, O'Colmain BJ, Munoz B, et al. Prevalence of agerelated macular degeneration in the United States. Arch Opbthalmol. 2004;122:564-572.

17. Varma R, Fraser-Bell S, Tan S, et al. Prevalence of age-related macular degeneration in Latinos: the Los Angeles Latino eye study. Opbthalmology. 2004;111:1288-1297.

18. Munoz B, Klein R, Rodriguez J, et al. Prevalence of age-related macular degeneration in a population-based sample of Hispanic people in Arizona: Proyecto VER. Arch Opbthalmol. 2005;123: 1575-1580.

19. Augood CA, Vingerling JR, de Jong PT, et al. Prevalence of agerelated maculopathy in older Europeans: the European Eye Study (EUREYE). Arch Ophthalmol. 2006;124:529-535.

20. Van Leeuwen R, Chakravarthy U, Vingerling H, et al. Grading of age-related maculopathy for epidemiological studies: is digital imaging as good as $35 \mathrm{~mm}$ film. Ophthalmology. 2003;110:15401544 . 